\title{
Graph Partitioning and Traffic Grooming with Bounded Degree Request Graph*
}

\author{
${\text { Zhentao } \mathrm{Li}^{1} \quad \text { Ignasi Sau }}^{2,3}$ \\ STUDENT PAPER
}

\begin{abstract}
We study a graph partitioning problem which arises from traffic grooming in optical networks. We wish to minimize the equipment cost in a SONET WDM ring network by minimizing the number of Add-Drop Multiplexers (ADMs) used. We consider the version introduced by Muñoz and Sau [12] where the ring is unidirectional with a grooming factor $C$, and we must design the network (namely, place the ADMs at the nodes) so that it can support any request graph with maximum degree at most $\Delta$. This problem is essentially equivalent to finding the least integer $M(C, \Delta)$ such that the edges of any graph with maximum degree at most $\Delta$ can be partitioned into subgraphs with at most $C$ edges and each vertex appears in at most $M(C, \Delta)$ subgraphs [12]. The cases where $\Delta=2$ and $\Delta=3, C \neq 4$ were solved by Muñoz and Sau [12]. In this article we establish the value of $M(C, \Delta)$ for many more cases, leaving open only the case where $\Delta \geq 5$ is odd, $\Delta(\bmod 2 C)$ is between 3 and $C-1, C \geq 4$, and the request graph does not contain a perfect matching. In particular, we answer a conjecture of [12].
\end{abstract}

Keywords: optical networks, traffic grooming, ADM, graph decomposition, cubic graph.

\section{Introduction}

Traffic grooming is the generic term for packing low rate signals into higher speed streams in optical networks $[4,7,11,15]$. By using traffic grooming, it is possible to bypass the electronics at the nodes which are not sources or destinations of traffic, and therefore reduce the cost of the network. Typically, in a Wavelength Division Multiplexing (WDM) network, instead of having one SONET Add Drop Multiplexer (ADM) on every wavelength at every node, it is possible to have ADMs only for the wavelengths used at that node; the other wavelengths being optically routed without electronic switching.

The so called traffic grooming problem consists of minimizing the total number of ADMs to be used, in order to reduce the overall cost of the network. The problem is easily seen to be NP-hard for an arbitrary set of requests in very simple topologies. In fact, hardness and approximation results exist for traffic grooming in ring, star, and tree networks $[2,8,9]$.

Here we consider unidirectional SONET/WDM ring networks with symmetric requests. In this case, the routing is unique and to each request between two nodes, we assign a wavelength and some bandwidth on this wavelength. If the traffic is uniform and any given wavelength can carry at most $C$ requests, we can assign at most $\frac{1}{C}$ of the bandwidth to each request. $C$ is known as the grooming factor. Furthermore, if the traffic requirement is symmetric, we may

\footnotetext{
*This work has been partially supported by: 1st author: NSERC; 2nd author: European project IST FET AEOLUS, PACA region of France, Ministerio de Ciencia e Innovación, European Regional Development Fund under project MTM2008-06620-C03-01/MTM, and Catalan Research Council under project 2005SGR00256.

${ }^{1}$ School of Computer Science - McGill University - Montreal, Canada. Email: zhentao.li@mail.mcgill.ca.

${ }^{2}$ Graph Theory and Combinatorics group - Applied Mathematics IV Department of UPC - Barcelona, Spain.

${ }^{3}$ Mascotte project - INRIA/CNRS/UNSA - Sophia Antipolis, France. Email: ignasi.sau@sophia.inria.fr.
} 
assume that symmetric requests are assigned the same wavelength, as it is easy to show (by exchanging wavelengths) that there exists an optimal solution where all symmetric requests are given the same wavelength. Then each pair of symmetric requests uses $\frac{1}{C}$ of the bandwidth in the whole ring. If the two end-nodes are $u$ and $v$, we need one ADM at node $u$ and one at node $v$. The main point is that if two requests have a common end-node, they can share an ADM if they are assigned the same wavelength.

The traffic grooming problem for a unidirectional SONET ring with $n$ nodes, grooming ratio $C$, and a symmetric request graph $R$ has been modeled as a graph partition problem as follows (see [3,10]). Each edge of $R$ corresponds to a pair of symmetric requests, and edges are colored by their assigned wavelength $\lambda$. All edges of color $\lambda$ induce a connected subgraph $B_{\lambda}$ of $R$, where each node corresponds to an ADM. The grooming constraint, i.e. the fact that a wavelength can carry at most $C$ requests, translates to an upper bound $C$ on the number of edges in each $B_{\lambda}$. The cost corresponds to the total number of vertices used in the subgraphs, and the objective is therefore to minimize $\sum_{\lambda}\left|V\left(B_{\lambda}\right)\right|$.

While most of previous work has focused on the case where the requests are given as input [2$4,7-11]$, we consider the case where only the network topology is given, together with a bound $\Delta$ on the request graph. We would like to place, for each value of the grooming factor $C$, a minimum number of ADMs at each node in such a way that they could support any traffic pattern where each node is the end-node of at most $\Delta$ requests. This model was recently introduced in [12], and it is interesting because the network can support dynamic traffic without replacement of the ADMs. The problem can be formulated as a graph partition problem as follows:

$\Delta$-Degree-Bounded Traffic Grooming in Unidirectional Rings

Input: Three integers $n$ (size of the ring), $C$ (grooming factor), and $\Delta$ (maximum degree).

Output: An assignment of $A(v)$ ADMs to each vertex $v$ of the ring, in such a way that for any request graph $G$ with maximum degree at most $\Delta$, there exists a partition of $E(G)$ into subgraphs $\left\{B_{\lambda}\right\}_{1 \leq \lambda \leq \Lambda}=\mathcal{B}$, such that:

(i) $\left|E\left(B_{\lambda}\right)\right| \leq C$ for all $\lambda$; and

(ii) each vertex $v \in V(G)$ appears in at most $A(v)$ subgraphs.

Objective: Minimize $\sum_{v} A(v)$.

The optimum to the above problem for each $n, C, \Delta$ is denoted by $A(n, C, \Delta)$. Given a graph with maximum degree at most $\Delta$, a partition of $G$ into subgraphs with at most $C$ edges is called a $C$-edge-partition of $G$.

Previous work and our contribution. The cases where $\Delta=2$ and the cases $\Delta=3$, $C \neq 4$ were solved in [12]. In this article we establish the value of $M(C, \Delta)$ for the following cases: when $\Delta=3$ and $C=4$ (answering a conjecture of [12], c.f. Section 3), when $\Delta \geq 4$ is even for any $C$ (c.f. Section 4), and when $\Delta \geq 5$ is odd (c.f. Section 5 ) and either $C \in\{2,3\}$, $\Delta(\bmod 2 C)=1, \Delta(\bmod 2 C) \geq C$, or the request graph contains a perfect matching. We provide a strengthening of one of our results when $\Delta \geq 5$ is odd in Appendix A. We first fix the notation below and give some preliminaries in Section 2.

Notation. The (multi)graphs considered in this paper are finite and without self-loops. Edges are denoted $\{u, v\}$. The degree of a vertex $v$ is the number of edges containing $v$ as an end-point. The maximum degree of a (multi)graph is the maximum degree over all its vertices. A $\Delta$-graph is a (multi)graph with maximum degree at most $\Delta$. $\mathcal{G}_{\Delta}$ denotes the class of all $\Delta$-graphs. A $\Delta$-regular (multi)graph is a graph in which all vertices have degree $\Delta$. An almost $\Delta$-regular 
(multi)graph is a (multi)graph in which all vertices have degree $\Delta$ except possibly one which has degree $\Delta-1$. A bridge in a (multi)graph $G$ is an edge whose removal disconnects $G$. A matching in a (multi)graph $G=(V, E)$ is a subset $M \subseteq E$ which contains each vertex at most once. A perfect matching is a matching containing all vertices. A digon is a cycle of length 2 . A trail in a (multi)graph is a sequence $\left\{\left\{x_{1}, x_{2}\right\},\left\{x_{2}, x_{3}\right\}, \ldots,\left\{x_{k-1}, x_{k}\right\}\right\}$ of distinct edges in which the second end of an edge is the first end of the next edge (the same pair of vertices may appear more than once if there is more than one edge between them). Vertices $x_{2}, x_{3}, \ldots, x_{k-1}$ of a trail are called midpoints. The length of a trail is the number of edges in it. Given a (multi)graph $G=(V, E)$ and a subset of vertices $V^{\prime} \subseteq V$, we denote by $G-V^{\prime}$ the (multi)graph obtained from $G$ by removing the vertices in $V^{\prime}$, the edges incident with vertices in $V^{\prime}$, and isolated vertices (if any). Similarly, given a subset of edges $E^{\prime} \subseteq E$, we denote by $G-E^{\prime}$ the (multi)graph obtained from $G$ by removing the edges in $E^{\prime}$ and isolated vertices (if any).

\section{Reducing the problem}

We begin by applying some easy reductions to the problem and recalling some results from [12] that will be used throughout. Let $M(C, \Delta)$ be the smallest number $M$ such that $A(n, C, \Delta) \leq$ $M n$ for all $n$. It is known that $M(C, \Delta)$ is an integer for all values of $C, \Delta$ [12]. If the request graph is further restricted to belong to a subclass of graphs $\mathcal{C} \subseteq \mathcal{G}_{\Delta}$, then the corresponding positive integer is denoted by $M(C, \Delta, \mathcal{C})$.

By the discussion above, $A(n, C, \Delta)$ is of the form $A(n, C, \Delta)=M(C, \Delta) n-\alpha(C, \Delta)$, where $M(C, \Delta)$ and $\alpha(C, \Delta)$ are integers depending only on $C$ and $\Delta$. Suppose that a $\Delta$-graph $H$ requires at least $M(C, \Delta)+1 \mathrm{ADMs}$ at some vertex. Since any $\Delta$-graph must be supported with the same ADMs, by relabeling the vertices of $H$ we could force at least $M(C, \Delta)+1 \mathrm{ADMs}$ in $\Omega(n)$ nodes of the network. This would contradict the definition of $M(C, \Delta)$. Therefore, each vertex can appear in at most $M(C, \Delta)$ subgraphs. So we may conclude the following.

Remark 2.1. For each value of $C$ and $\Delta, \Delta$-Degree-Bounded Traffic Grooming in UNIDIRECTIONAL RINGS reduces to finding the least integer $M(C, \Delta)$ such that the edges of any $\Delta$-graph can be partitioned into subgraphs with at most $C$ edges and each vertex appears in at most $M(C, \Delta)$ subgraphs.

This allows us to give an equivalent definition of $M(C, \Delta)$. Let $G \in \mathcal{G}_{\Delta}$ and let $\mathcal{P}_{C}(G)$ be the set of $C$-edge-partitions of $G$. For $P \in \mathcal{P}_{C}(G)$, let occ $(P)$ be the maximum number of occurrences of a vertex in the partition, that is,

$$
\operatorname{occ}(P)=\max _{v \in V(G)}\left|\left\{B_{\lambda} \in P: v \in B_{\lambda}\right\}\right|, \quad \text { and then } \quad M(C, \Delta)=\max _{G \in \mathcal{G}_{\Delta}}\left(\min _{P \in \mathcal{P}_{C}(G)} \operatorname{occ}(P)\right) .
$$

In the remainder of this paper, we use Remark 2.1 and focus on determining $M(C, \Delta)$ for each value of $C$ and $\Delta$. Observe also that any $\Delta$-graph $H$ is a subgraph of some $\Delta$-regular graph $G$ (with possibly more vertices). Note also that if we restrict a partition of $G$ to the vertices of $H$, the number of occurrences of the vertices cannot increase. Therefore,

Remark 2.2. $M(C, \Delta)=M(C, \Delta, \mathcal{C})$, where $\mathcal{C}$ is the class of $\Delta$-regular graphs.

The following two results will be used throughout the article.

Lemma 2.3 (Muñoz and Sau [12]). The following statements hold trivially:

(i) $M(C, 1)=1$ for all $C \geq 1$.

(ii) $M(1, \Delta)=\Delta$ for all $\Delta \geq 1$. 
(iii) If $C^{\prime} \geq C$, then $M\left(C^{\prime}, \Delta\right) \leq M(C, \Delta)$.

(iv) If $\Delta^{\prime} \geq \Delta$, then $M\left(C, \Delta^{\prime}\right) \geq M(C, \Delta)$.

(v) $M(C, \Delta) \leq \Delta$ for all $C, \Delta \geq 1$.

Proposition 2.4 (Muñoz and Sau [12]). $M(C, \Delta) \geq\left\lceil\frac{C+1}{C} \frac{\Delta}{2}\right\rceil$ for all values of $C, \Delta$.

In [12] it is proved that $M(C, 2)=2$ for any $C \geq 1$, that $M(C, 3)=3$ for $C \leq 3$, and that $M(C, 3)=2$ for $C \geq 5$. The latter result was proved using a result of Thomassen [14], settling a conjecture of Bermond et al. [5], stating that the edges of a cubic graph can be 2-colored such that each monochromatic component is a path of length at most 5 .

Let us now discuss how these ideas can be extended to other values of $C, \Delta$. A linear $C$ forest in a graph is a forest consisting of paths of length at most $C$. The linear $C$-arboricity of a graph $G$ is the minimum number of linear $C$-forests required to partition $E(G)$, and is denoted by $l a_{C}(G)[5]$. Let $l a_{C}(\Delta)=\max _{G \in \mathcal{G}_{\Delta}} l a_{C}(G)$. Clearly $M(C, \Delta) \leq l a_{C}(\Delta)$ for all $C, \Delta$, since the paths in a linear $C$-forest are graphs with at most $C$ edges. Therefore, the following upper bound given by Alon et al. [1] also applies to $M(C, \Delta)$.

Theorem 2.5 (Alon et al. [1]). There is an absolute constant $\beta>0$ such that for $\sqrt{\Delta}>C \geq 2$,

$$
l a_{C}(\Delta) \leq \frac{C+1}{C} \frac{\Delta}{2}+\beta \sqrt{C \Delta \log \Delta} .
$$

The first term of the right-hand side of Equation (1) is equal to the lower bound of Proposition 2.4, so Theorem 2.5 provides an additive $\mathcal{O}(\sqrt{C \Delta \log \Delta})$-approximation of $M(C, \Delta)$ for $\sqrt{\Delta}>C \geq 2$. We improve this bound for $M(C, \Delta)$ in Sections 4 and 5 (without using the linear $C$-arboricity), providing an additive 1-approximation of $M(C, \Delta)$ for any value of $C$ and $\Delta$, which is optimal for any even $\Delta$, and in many cases for odd $\Delta$.

\section{Case $\Delta=3, C=4$}

Muñoz and Sau conjectured that $M(4,3)=2$ [12], which we now prove. We first need the following classical result.

Theorem 3.1 (Petersen [13]). Any cubic bridgeless graph has a perfect matching.

Theorem 3.1 can be easily extended to cubic bridgeless multigraphs.

Corollary 3.2. Any cubic bridgeless multigraph without self-loops has a perfect matching.

Proof. Let $G$ be a cubic multigraph without self-loops. We can assume that $G$ has no triple edges, otherwise $G$ has only 2 vertices and any of the 3 edges is a perfect matching. Consider the simple graph $G^{\prime}$ built from $G$ as follows: for each digon $\{\{u, v\},\{u, v\}\}$, add 2 new vertices $s_{u v}$ and $t_{u v}$, and replace the digon with the edges $\left\{u, s_{u v}\right\},\left\{u, t_{u v}\right\},\left\{v, s_{u v}\right\},\left\{v, t_{u v}\right\}$, and $\left\{s_{u v}, t_{u v}\right\}$. By Theorem 3.1, $G^{\prime}$ has a perfect matching $M^{\prime}$. We now construct a perfect matching $M$ of $G$ from $M^{\prime}$. For each edge $e \in M^{\prime}$ such that $e$ was also an edge of $G$, put $e$ in $M^{\prime}$. For each digon $\{\{u, v\},\{u, v\}\}$ of $G$, if any of the pairs $\left\{\left\{u, s_{u v}\right\},\left\{v, t_{u v}\right\}\right\}$ or $\left\{\left\{u, t_{u v}\right\},\left\{v, s_{u v}\right\}\right\}$ is in $M^{\prime}$, put one of the copies of $\{u, v\}$ in $M$. Otherwise, $\left\{s_{u v}, t_{u v}\right\}$ belongs to $M^{\prime}$ and we do nothing. It is easy to check that $M$ is a perfect matching of $G$.

We are ready to prove the main result of this section.

Theorem 3.3. The edges of every almost 3-regular multigraph $G$ without self-loops can be partitioned into a set $\mathcal{W}=\left\{W_{1}, W_{2}, \ldots, W_{k}\right\}$ of trails of length at most 4 such that each vertex appears as the midpoint of a trail. 
Proof. Suppose the theorem is false and let $G$ be a counterexample with the minimum number of vertices. $G$ is connected as otherwise, we can take the union of the partitions of its connected components, which exist by minimality of $G$.

Suppose first that $G$ contains a bridge $e=\{u, v\}$. Then $G-\{e\}$ has exactly two components: $U$ containing $u$ and $V$ containing $v$. Without loss of generality, we may choose $U$ to be the component with no degree 2 vertex in $G$ and $e$ is chosen so that $U$ is maximal with this property. Thus this component $U$ of $G-\{e\}$ is almost 3-regular (only $u$ has degree 2). By minimality of $G, U$ can be partitioned into a set $\mathcal{W}^{u}$ of trails as in the statement of the theorem.

If $v$ has degree 2 in $G$ then $V-\{v\}$ is almost 3-regular. By minimality of $G, V-\{v\}$ can be partitioned into a set $\mathcal{W}^{v}$ of trails as in the theorem. Now the only edges of $G$ not in any trail in $\mathcal{W}^{u} \cup \mathcal{W}^{v}$ are those incident to $v$. Thus taking $\mathcal{W}^{u} \cup \mathcal{W}^{v}$ together with a trail consisting of the 2 edges incident to $v$ (which has $v$ as a midpoint) yields the required partition of the edges of $G$ into trails. This contradicts the fact that $G$ is a counterexample.

If $v$ has degree 3 in $G$, let $x, y$ be the neighbors of $v$ in $V$ (see Figure 1(a)). We can assume $x \neq y$ (i.e., $\{v, x\}$ and $\{v, y\}$ are not parallel edges) since otherwise, the third edge incident to $x=y$ is a cut edge whose choice (instead of $e$ ) would increase the size of $U$. Let $H$ be the graph obtained from $V-\{v\}$ by adding an edge $f=\{x, y\}$ (see Figure 1(b)). By minimality of $G$, $H$ can be partitioned into a set $\mathcal{W}^{v}$ of trails. We now attempt to transform $\mathcal{W}^{u} \cup \mathcal{W}^{v}$ into a partition of $G$ into trails.

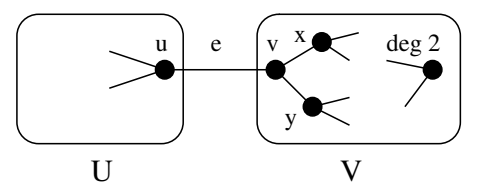

(a)

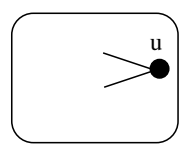

$\mathrm{W}^{\mathrm{u}}$

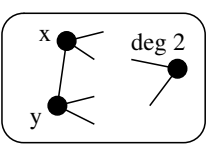

$\mathrm{W}^{\mathrm{v}}$

(b)

Figure 1: (a) A bridge $e=\{u, v\}$ in an almost 3-regular graph $G$ with components $U$ and $V$ of $G-\{e\}$. (b) Graphs smaller than $G$ from which we obtain a partition into trails $W^{u}$ and $W^{v}$.

The edge $f$ appears in some trail $\left\{W_{1},\{x, y\}, W_{2}\right\}$ of $\mathcal{W}^{v}$, where $W_{1}$ is a (possibly empty) trail ending at $x$ and $W_{2}$ is a (possibly empty) trail starting at $y$. At least one of the subtrails $\left\{W_{1},\{x, y\}\right\}$ or $\left\{\{x, y\}, W_{2}\right\}$ has fewer than 3 edges. Without loss of generality, it is $\left\{W_{1},\{x, y\}\right\}$. Replace this trail with $\left\{W_{1},\{x, v\},\{v, u\}\right\}$ which has length at most 4 , and $\left\{\{v, y\}, W_{2}\right\}$ which has length less than or equal to $\left\{W_{1},\{x, y\}, W_{2}\right\}$. Note that $x$ and $v$ are midpoints of the first trail and $y$ is the midpoint of the second trail. Furthermore, any other vertex which was a midpoint in $\left\{W_{1},\{x, y\}, W_{2}\right\}$ is still a midpoint (since $W_{1}$ and $W_{2}$ appear as subtrails).

Thus the union of $\mathcal{W}^{u}$ and $\mathcal{W}^{v}$ with the above replacement yields a partition of $G$ into trails of length at most 4 with the desired property, which is a contradiction.

We may now assume that $G$ contains no bridges. If $G$ is 3 -regular, let $G^{\prime}=G$. Otherwise, let $G^{\prime}$ be the graph obtained from $G$ by replacing the vertex of degree 2 with an edge between its endpoints. Note that $G^{\prime}$ is 3-regular and contains no bridges. Therefore, by Corollary 3.2, $G^{\prime}$ contains a perfect matching $M \subseteq E\left(G^{\prime}\right)$.

Since $G^{\prime}$ is 3-regular, $G^{\prime}-M$ is 2-regular. Thus, $G^{\prime}-M$ is a union of disjoint cycles. We can orient the cycles of $G^{\prime}-M$ so that each vertex $v$ has exactly one edge $e_{v}$ pointing towards $v$. For each edge $\{u, v\} \in M, W_{u v}=\left\{e_{u},\{u, v\}, e_{v}\right\}$ is a trail of length 3 (see Figure 2). Note that $\mathcal{W}=\left\{W_{u v} \mid\{u, v\} \in M\right\}$ is a partition of the edges of $G^{\prime}$ into trails of length 3. Furthermore, every vertex $u$ in the matching appears as the midpoint of the trail corresponding to the edge of the matching in which $u$ appears. Since $M$ is a perfect matching, every vertex appears as the midpoint of some trail in $\mathcal{W}$. Thus $G^{\prime} \neq G$ as otherwise, we have constructed a partition 


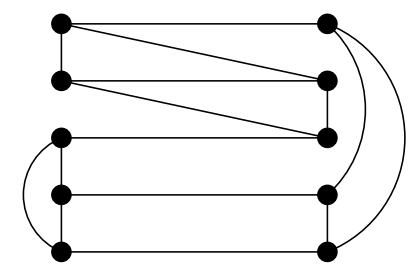

(a)

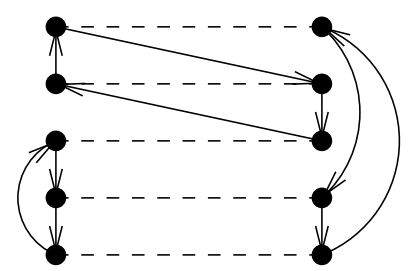

(b)

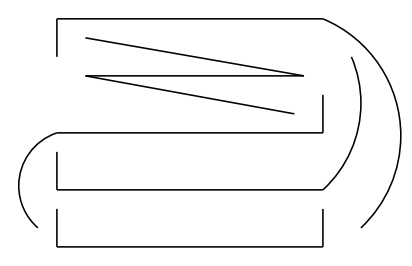

(c)

Figure 2: (a) A 3-regular graph $G^{\prime}$ with no bridges. (b) A matching $M$ of $G^{\prime}$ (shown in dashed lines) and an orientation of the cycles of $G^{\prime}-M$. (c) A partition of the edges of $G^{\prime}$ into trails of length 3 using $M$ and the orientation of the cycle of $G^{\prime}-M$ in (b).

as required by the theorem. So $G$ has a vertex $v$ of degree 2 which we replaced with an edge $e=\{x, y\}$ to obtain $G^{\prime}$. Let $W=\left\{W_{1},\{x, y\}, W_{2}\right\}$ be the trail in $\mathcal{W}$ containing $e$, and recall that $W$ has length 3 . Replacing $W$ with $\left\{W_{1},\{x, v\},\{v, y\}, W_{2}\right\}$ in $\mathcal{W}$ yields a partition of $E(G)$ into trails of length at most 4 , which is a contradiction.

Note that the simple trees with some vertex of degree 3 and the digon with a pendant edge at each side are not allowed in the partition stated in Theorem 3.3, since these graphs cannot be thought of as trails. The following corollary answers the conjecture of [12].

Corollary 3.4. $M(4,3)=2$.

Proof. By Remark 2.2, we may restrict ourselves to 3-regular graphs. Thus, a 3-regular graph $G$ is almost 3-regular and we may apply Theorem 3.3 to obtain a partition $\mathcal{W}$. Let $\mathcal{B}=$ $\{E(W)\}_{W \in \mathcal{W}}$. Each vertex of $G$ appears in at most two elements of $\mathcal{B}$, as $G$ is 3-regular and each vertex appears as the midpoint of some trail in $\mathcal{W}$.

\section{Case $\Delta \geq 4$ even}

In this section we establish the value of $M(C, \Delta)$ for $\Delta \geq 4$ even and any value of $C$.

Theorem 4.1. Let $\Delta \geq 4$ be even. Then for any $C \geq 1, M(C, \Delta)=\left\lceil\frac{C+1}{C} \frac{\Delta}{2}\right\rceil$.

Proof. The lower bound follows from Proposition 2.4. Let us give an explicit construction for any $\Delta$-regular graph $G=(V, E)$. Orient the edges of $G$ in an Eulerian tour, and assign to each vertex $v \in V$ its $\Delta / 2$ out-edges, namely $E_{v}^{+}$. For each $v \in V$, partition $E_{v}^{+}$into $\left\lceil\frac{\Delta}{2 C}\right\rceil$ stars with $C$ edges centered at $v$ (except, possibly, one star with fewer edges). Each vertex $v$ appears as a leaf in stars centered at other vertices exactly $\Delta-\Delta / 2=\Delta / 2$ times. Therefore, the number of occurrences of each vertex in this partition is

$$
\left\lceil\frac{\Delta}{2 C}\right\rceil+\frac{\Delta}{2}=\left\lceil\frac{\Delta}{2}\left(1+\frac{1}{C}\right)\right\rceil=\left\lceil\frac{C+1}{C} \frac{\Delta}{2}\right\rceil .
$$

\section{Case $\Delta \geq 5$ odd}

The cases where $\Delta$ is odd turn out to be inherently much more complicated than the cases where $\Delta$ is even. In Section 5.1, we present a general construction which differs from the lower bound of Proposition 2.4 by at most 1 , and we determine when this construction is optimal. In Section 5.2, we present an optimal construction for graphs with a perfect matching. Finally, in Section 5.3, we provide an improved lower bound when $\Delta \equiv C(\bmod 2 C)$, which meets our upper bound. 


\subsection{General upper bound}

The following proposition provides a general upper bound, which differs from the lower bound of Proposition 2.4 by at most 1 .

Proposition 5.1. Let $\Delta \geq 5$ be odd. Then for any $C \geq 1, M(C, \Delta) \leq\left\lceil\frac{C+1}{C} \frac{\Delta}{2}+\frac{C-1}{2 C}\right\rceil$.

Proof. Let $G$ be a $\Delta$-regular graph. Since $\Delta$ is odd, $|V(G)|$ is even. Add a perfect matching $M$ to $G$ to obtain a $(\Delta+1)$-regular multigraph $G^{\prime}$. Orient the edges of $G^{\prime}$ in an Eulerian tour, and assign to each vertex $v \in V\left(G^{\prime}\right)$ its $(\Delta+1) / 2$ out-edges $E_{v}^{+}$. Remove the edges of $M$ and, as in the case $\Delta$ even, partition $E_{v}^{+}$into stars with at most $C$ edges. To count the number of occurrences of each vertex, we distinguish two cases. If an edge of $M$ is in $E_{v}^{+}$, then $v$ appears as center in $\left\lceil\frac{\Delta-1}{2 C}\right\rceil$ stars and as a leaf in $\Delta-\frac{\Delta-1}{2}$ stars. Summing both terms yields

$$
\left\lceil\frac{\Delta-1}{2 C}\right\rceil+\Delta-\frac{\Delta-1}{2}=\left\lceil\frac{C+1}{C} \frac{\Delta}{2}+\frac{C-1}{2 C}\right\rceil .
$$

Otherwise, if no edge of $M$ is in $E_{v}^{+}$, the number of occurrences of $v$ is

$$
\left\lceil\frac{\Delta+1}{2 C}\right\rceil+\Delta-\frac{\Delta+1}{2}=\left\lceil\frac{C+1}{C} \frac{\Delta}{2}+\frac{1-C}{2 C}\right\rceil \leq\left\lceil\frac{C+1}{C} \frac{\Delta}{2}+\frac{C-1}{2 C}\right\rceil .
$$

The upper bound of Proposition 5.1 and the lower bound of Proposition 2.4 are equal for, roughly speaking, half of the pairs $C, \Delta$, as shown in the following corollary.

Corollary 5.2. Let $\Delta \geq 5$ be odd. If $\Delta(\bmod 2 C)=1$ or $\Delta(\bmod 2 C) \geq C+1$, then $M(C, \Delta)=\left\lceil\frac{C+1}{C} \frac{\Delta}{2}\right\rceil$.

Proof. Let $\Delta=\lambda \cdot 2 C+h$, with $h$ odd, $1 \leq h \leq 2 C-1$. Writing $k:=\lambda(C+1)+\frac{h-1}{2}$, the lower bound of Proposition 2.4 equals $k+\left\lceil\frac{1}{2}+\frac{h}{2 C}\right\rceil$, and the upper bound of Proposition 5.1 equals $k+\left\lceil 1+\frac{h-1}{2 C}\right\rceil$. If $h=1$ both bounds equal $k+1$, and if $h \geq C+1$ both bounds equal $k+2$.

In particular, when $C=2$ and $\Delta$ is odd, $\Delta(\bmod 2 C)$ is either 1 or 3 , and then by Corollary 5.2 the lower bound is attained, as stated in the following corollary.

Corollary 5.3. For any $\Delta \geq 5$ odd, $M(2, \Delta)=\left\lceil\frac{3 \Delta}{4}\right\rceil$.

We shall see in Theorem 5.5 that if $\Delta \equiv C(\bmod 2 C)$, then $M(C, \Delta)=\left\lceil\frac{C+1}{C} \frac{\Delta}{2}\right\rceil+1$.

\subsection{Optimal construction for graphs with a perfect matching}

If the input graph has a perfect matching, we can prove that the lower bound is attained for all values of $C$.

Proposition 5.4. Let $\Delta \geq 5$ be odd and let $\mathcal{C}$ be the class of $\Delta$-regular graphs than contain a perfect matching. Then $M(C, \Delta, \mathcal{C})=\left\lceil\frac{C+1}{C} \frac{\Delta}{2}\right\rceil$.

Proof. First, it is easy to check that the proof of the lower bound of Proposition 2.4 in [12] still carries over when restricted to the class of graphs with a perfect matching. To prove the upper bound, if $G$ is $\Delta$-regular with a perfect matching $M$, we orient the edges of $G-M$ in an Eulerian tour, and assign to each vertex $v \in V(G)$ its $\frac{\Delta-1}{2}$ out-edges $E_{v}^{+}$. We distinguish three cases. 
(1) $\Delta<C$. For each edge $\{u, v\} \in M$, build the tree with $\Delta$ edges consisting of $\{u, v\}, \frac{\Delta-1}{2}$ edges from $E_{u}^{+}$, and $\frac{\Delta-1}{2}$ edges from $E_{v}^{+}$. The number of occurrences of each vertex is $1+\Delta-\frac{\Delta+1}{2}=\frac{\Delta+1}{2}$. The lower bound equals $\left\lceil\frac{C+1}{C} \frac{\Delta}{2}\right\rceil=\frac{\Delta-1}{2}+\left\lceil\frac{1}{2}+\frac{\Delta}{2 C}\right\rceil$, which equals $\frac{\Delta+1}{2}$ as $\Delta<C$.

(2) $\Delta \geq C$ and $C \geq 3$ is odd (the case $C=1$ is trivial by Lemma 2.3). For each edge $\{u, v\} \in M$, build the tree with $C$ edges consisting of $\{u, v\}, \frac{C-1}{2}$ edges from $E_{u}^{+}$, and $\frac{C-1}{2}$ edges from $E_{v}^{+}$. Partition the remaining $\frac{\Delta-1}{2}-\frac{C-1}{2}=\frac{\Delta-C}{2}$ edges assigned to each vertex into $\left\lceil\frac{\Delta-C}{2 C}\right\rceil$ stars with at most $C$ edges. The number of occurrences of each vertex is

$$
1+\left\lceil\frac{\Delta-C}{2 C}\right\rceil+\Delta-\frac{\Delta+1}{2}=\left\lceil\frac{C+1}{C} \frac{\Delta}{2}\right\rceil .
$$

(3) $\Delta \geq C$ and $C \geq 4$ is even (the case $C=2$ is solved by Corollary 5.3). Build the tree with $C-1$ edges consisting of $\{u, v\}, \frac{C-2}{2}$ edges from $E_{u}^{+}$, and $\frac{C-2}{2}$ edges from $E_{v}^{+}$. Partition the remaining $\frac{\Delta-1}{2}-\frac{C-2}{2}=\frac{\Delta-C+1}{2}$ edges assigned to each vertex into stars with at most $C$ edges. The number of occurrences of each vertex is

$$
1+\left\lceil\frac{\Delta-C+1}{2 C}\right\rceil+\frac{\Delta-1}{2}=\left\lceil\frac{\Delta(C+1)+1}{2 C}\right\rceil=\left\lceil\frac{C+1}{C} \frac{\Delta}{2}\right\rceil,
$$

where the last equality holds because both $\Delta$ and $(C+1)$ are odd.

\subsection{Improved lower bounds}

In this section we prove a new lower bound which strictly improves on Proposition 2.4 when $\Delta \equiv C(\bmod 2 C)$. The idea is to generalize the counterexample given in [12, Proposition 4$]$ to prove that $M(3,3)=3$.

Theorem 5.5. Let $\Delta \geq 5$ be odd and let $L B(C, \Delta)=\left\lceil\frac{C+1}{C} \frac{\Delta}{2}\right\rceil$, the lower bound of Proposition 2.4. If $\Delta \equiv C(\bmod 2 C)$, then $M(C, \Delta)=L B(C, \Delta)+1$.

Proof. We prove that if $\Delta=k C$ with $k$ odd, then $M(C, \Delta) \geq \operatorname{LB}(C, \Delta)+1$ and thus, by Proposition 5.1, $M(C, \Delta)$ is equal to $\operatorname{LB}(C, \Delta)+1$. Since both $\Delta$ and $k$ are odd, so is $C$, and therefore $\operatorname{LB}(C, \Delta)=k \cdot \frac{C+1}{2}$.

We proceed to build a $\Delta$-regular graph $G$ with no $C$-edge-partition where each vertex is incident to at most $\operatorname{LB}(C, \Delta)$ subgraphs, hence implying that $M(C, \Delta)>\operatorname{LB}(C, \Delta)$. First, we construct a graph $H$ where all vertices have degree $\Delta$ except one which has degree $\Delta-1$. Furthermore, we build $H$ so that it has girth strictly greater than $C$. $H$ exists by [6]. Make $\Delta$ copies of $H$ and add a cut-vertex $v$ joined to all vertices of degree $\Delta-1$ to make our $\Delta$-regular graph $G$.

Now suppose for the sake of contradiction that there is a $C$-edge-partition $\mathcal{B}$ of $G$ where each vertex is incident to at most $\operatorname{LB}(C, \Delta)$ subgraphs. Since the girth of $G$ is greater than $C$, all the subgraphs in $\mathcal{B}$ are trees. Since $L B(C, \Delta)<\Delta, v$ must have degree at least 2 in some subgraph $T^{\prime} \in \mathcal{B}$. Since $\left|E\left(T^{\prime}\right)\right| \leq C$, the tree $T^{\prime}$ contains at most $\left\lfloor\frac{C-2}{2}\right\rfloor=\frac{C-3}{2}$ edges of a copy $H^{\prime}$ of $H$ intersecting $T^{\prime}$. Now we only work in $H^{\prime}$. Let $\alpha=\left|E\left(T^{\prime} \cap H^{\prime}\right)\right| \leq \frac{C-3}{2}$.

Let $\mathcal{B}^{\prime}=\left\{B \cap H^{\prime}\right\}_{B \in\left(\mathcal{B}-\left\{T^{\prime}\right\}\right)}$, with the empty subgraphs removed. That is, $\mathcal{B}^{\prime}$ contains the subgraphs in $\mathcal{B}$ that partition the edges in $H^{\prime}$ that are not in $T^{\prime}$. Let $n=\left|V\left(H^{\prime}\right)\right|$, which is odd as in $H^{\prime}$ there is one vertex of degree $\Delta-1$ and all the others have degree $\Delta$. Therefore, the total number of edges of the trees in $\mathcal{B}^{\prime}$ is

$$
\sum_{T \in \mathcal{B}^{\prime}}|E(T)|=\left|E\left(H^{\prime}\right)\right|-\alpha=\frac{n \Delta-1}{2}-\alpha=\frac{n k C-1}{2}-\alpha .
$$


As $\alpha \leq \frac{C-3}{2}$, from Equation (2) we get

$$
\sum_{T \in \mathcal{B}^{\prime}}|E(T)| \geq \frac{n k C-1}{2}-\frac{C-3}{2}=\left(\frac{n k-1}{2}\right) \cdot C+1 .
$$

As each tree in $\mathcal{B}^{\prime}$ has at most $C$ edges, from Equation (3) we get that $\left|\mathcal{B}^{\prime}\right|$, the number of trees in $\mathcal{B}^{\prime}$, satisfies

$$
\left|\mathcal{B}^{\prime}\right| \geq\left\lceil\frac{n k-1}{2}+\frac{1}{C}\right\rceil=\frac{n k-1}{2}+\left\lceil\frac{1}{C}\right\rceil=\frac{n k-1}{2}+1
$$

Clearly, the total number of vertices in the trees in $\mathcal{B}^{\prime}$ is exactly the total number of edges in the trees in $\mathcal{B}^{\prime}$ plus the number of trees in $B^{\prime}$, that is, $\sum_{T \in \mathcal{B}^{\prime}}|V(T)|=\sum_{T \in \mathcal{B}^{\prime}}|E(T)|+\left|\mathcal{B}^{\prime}\right|$. On the other hand, the tree $T^{\prime}$ contains $\alpha+1$ vertices of $H^{\prime}$, that is, $\mid V\left(T^{\prime} \cap H^{\prime} \mid=\alpha+1\right.$. Therefore, using Equations (2) and (4), we get that the total number of occurrences of the vertices in $H^{\prime}$ in some tree of $\mathcal{B}$ is

$$
\begin{aligned}
\sum_{v \in V\left(H^{\prime}\right)}|\{T \in \mathcal{B}: v \in T\}| & =\sum_{T \in \mathcal{B}^{\prime}}|V(T)|+\left|V\left(T^{\prime} \cap H^{\prime}\right)\right|=\sum_{T \in \mathcal{B}^{\prime}}|E(T)|+\left|\mathcal{B}^{\prime}\right|+\alpha+1 \\
& =\frac{n k C-1}{2}-\alpha+\left|\mathcal{B}^{\prime}\right|+\alpha+1 \geq \frac{n k C-1}{2}+\frac{n k-1}{2}+1+1 \\
& =n k \cdot \frac{C+1}{2}+1=n \cdot \operatorname{LB}(C, \Delta)+1,
\end{aligned}
$$

which implies that at least one vertex of $H^{\prime}$ appears in at least $\operatorname{LB}(C, \Delta)+1$ subgraphs, which is a contradiction to $\mathcal{B}$ being a $C$-edge-partition of $G$ in which each vertex appears in at most $\mathrm{LB}(C, \Delta)$ subgraphs. The theorem follows.

It turns out that Theorem 5.5 allows us to find the value of $M(3, \Delta)$ for any $\Delta \geq 5$ odd.

Corollary 5.6. For any $\Delta \geq 5$ odd, $M(3, \Delta)=\left\lceil\frac{2 \Delta+1}{3}\right\rceil$.

Proof. If $\Delta \equiv 1(\bmod 6)$ or $\Delta \equiv 5(\bmod 6)$, then by Corollary $5.2, M(3, \Delta)=\left\lceil\frac{2 \Delta}{3}\right\rceil=\left\lceil\frac{2 \Delta+1}{3}\right\rceil$. Otherwise, if $\Delta \equiv 3(\bmod 6)$, then by Theorem $5.5, M(3, \Delta)=\left\lceil\frac{2 \Delta}{3}\right\rceil+1=\left\lceil\frac{2 \Delta+1}{3}\right\rceil$.

\section{Conclusions}

We considered the traffic grooming problem in unidirectional WDM rings when the request graph belongs to the class of graphs with maximum degree $\Delta$. This problem is essentially equivalent to finding the least integer $M(C, \Delta)$ such that the edges of any graph with maximum degree at most $\Delta$ can be partitioned into subgraphs with at most $C$ edges and each vertex appears in at most $M(C, \Delta)$ subgraphs. We established the value of $M(C, \Delta)$ for many cases, leaving open only the case where $\Delta \geq 5$ is odd, $\Delta(\bmod 2 C)$ is between 3 and $C-1, C \geq 4$, and the graph does not contain a perfect matching. Table 1 summarizes what is known about $M(C, \Delta)$, including the case where the graph has a perfect matching.

For the remaining cases, we hope to either extend the counterexample given in Section 5.3 or to complete the partial proof given in Appendix A, which can be seen as a strengthening of Proposition 5.4. 


\begin{tabular}{|l||c|c|c|c|c|c|c|c|c|c|c|c|}
\hline$C \mid \Delta$ & 1 & 2 & 3 & 4 & 5 & 6 & 7 & 8 & 9 & $\ldots$ & $\Delta$ even & $\Delta$ odd \\
\hline \hline 1 & 1 & 2 & 3 & 4 & 5 & 6 & 7 & 8 & 9 & $\ldots$ & $\Delta$ & $\Delta$ \\
\hline 2 & 1 & 2 & 3 & 3 & 4 & 5 & 6 & 6 & 7 & $\ldots$ & $\left\lceil\frac{3 \Delta}{4}\right\rceil$ & $\left\lceil\frac{3 \Delta}{4}\right\rceil$ \\
\hline 3 & 1 & 2 & $3(2)$ & 3 & 4 & $5(4)$ & 5 & 6 & $7(6)$ & $\ldots$ & {$\left[\frac{2 \Delta}{3}\right\rceil$} & $\left\lceil\frac{2 \Delta+1}{3}\right\rceil\left(\left\lceil\frac{2 \Delta}{3}\right\rceil\right)$ \\
\hline 4 & 1 & 2 & 2 & 3 & 4 & 4 & 5 & 5 & 6 & $\ldots$ & $\left.\frac{5 \Delta}{8}\right\rceil$ & $\geq\left\lceil\frac{5 \Delta}{8}\right\rceil(=)$ \\
\hline 5 & 1 & 2 & 2 & 3 & $4(3)$ & 4 & 5 & 5 & 6 & $\ldots$ & $\left\lceil\frac{3 \Delta}{5}\right\rceil$ & $\geq\left\lceil\frac{3 \Delta}{5}\right\rceil(=)$ \\
\hline 6 & 1 & 2 & 2 & 3 & $\geq \mathbf{3}(=)$ & 4 & 5 & 5 & 6 & $\ldots$ & {$\left[\frac{7 \Delta}{12}\right\rceil$} & $\geq\left\lceil\frac{7 \Delta}{12}\right\rceil(=)$ \\
\hline 7 & 1 & 2 & 2 & 3 & $\geq \mathbf{3}(=)$ & 4 & $5(4)$ & 5 & 6 & $\ldots$ & $\left.\frac{4 \Delta}{7}\right\rceil$ & $\geq\left\lceil\frac{4 \Delta}{7}\right\rceil(=)$ \\
\hline 8 & 1 & 2 & 2 & 3 & $\geq \mathbf{3}(=)$ & 4 & $\geq 4(=)$ & 5 & 6 & $\ldots$ & $\left\lceil\frac{9 \Delta}{16}\right\rceil$ & $\geq\left\lceil\frac{9 \Delta}{16}\right\rceil(=)$ \\
\hline 9 & 1 & 2 & 2 & 3 & $\geq \mathbf{3}(=)$ & 4 & $\geq 4(=)$ & 5 & $6(5)$ & $\ldots$ & $\left\lceil\frac{5 \Delta}{9}\right\rceil$ & $\geq\left\lceil\frac{5 \Delta}{9}\right\rceil(=)$ \\
\hline$\ldots$ & $\ldots$ & $\ldots$ & $\ldots$ & $\ldots$ & $\ldots$ & $\ldots$ & $\ldots$ & $\ldots$ & $\ldots$ & $\ldots$ & $\ldots$ & $\ldots$ \\
\hline$C$ & 1 & 2 & 2 & 3 & $\geq \mathbf{3}(=)$ & 4 & $\geq \mathbf{4}(=)$ & 5 & $\geq \mathbf{5}(=)$ & $\ldots$ & $\left\lceil\frac{C+1}{C} \frac{\Delta}{2}\right\rceil$ & $\geq\left\lceil\frac{\mathrm{C}+1}{\mathrm{C}} \frac{\Delta}{2}\right\rceil(=)$ \\
\hline
\end{tabular}

Table 1: Known values of $M(C, \Delta)$. The red bold cases remain open. The (blue) cases in brackets only hold if the graph has a perfect matching. The symbol "(=)" means that the corresponding lower bound is attained.

\section{References}

[1] N. Alon, V. Teague, and N. C. Wormald. Linear Arboricity and Linear k-Arboricity of Regular Graphs. Graphs and Combinatorics, 17(1):11-16, 2001.

[2] O. Amini, S. Pérennes, and I. Sau. Hardness and Approximation of Traffic Grooming. In 18th International Symposium on Algorithms and Computation (ISAAC), volume 4835 of LNCS, pages 561-573, 2007.

[3] J.-C. Bermond and D. Coudert. Traffic Grooming in Unidirectional WDM Ring Networks using Design Theory. In IEEE ICC, volume 2, pages 1402-1406, 2003.

[4] J.-C. Bermond and D. Coudert. The CRC Handbook of Combinatorial Designs (2nd edition), volume 42 of Discrete Mathematics and its Applications, chapter VI.27, Grooming, pages 493-496. CRC Press, C.J. Colbourn and J.H. Dinitz edition, 2006.

[5] J.-C. Bermond, J.-L. Fouquet, M. Habib, and B. Péroche. On linear k-arboricity. Discrete Mathematics, $52(2-3): 123-132,1984$.

[6] L. S. Chandran. A high girth graph construction. SIAM J. Discrete Math, 16(3):366-370, 2003.

[7] R. Dutta and N. Rouskas. Traffic grooming in WDM networks: Past and future. IEEE Network, 16(6):46-56, 2002.

[8] M. Flammini, G. Monaco, L. Moscardelli, M. Shalom, and S. Zaks. Approximating the traffic grooming problem in tree and star networks. Journal of Parallel and Distributed Computing, 68(7):939-948, 2008.

[9] M. Flammini, L. Moscardelli, M. Shalom, and S. Zaks. Approximating the traffic grooming problem. Journal of Discrete Algorithms, 6(3):472-479, 2008.

[10] O. Goldschmidt, D. Hochbaum, A. Levin, and E. Olinick. The SONET edge-partition problem. Networks, 41(1):13-23, 2003.

[11] E. Modiano and P. Lin. Traffic grooming in WDM networks. IEEE Communications Magazine, 39(7):124$129,2001$.

[12] X. Muñoz and I. Sau. Traffic Grooming in Unidirectional WDM Rings with Bounded Degree Request Graph. In 34th International Workshop on Graph-Theoretic Concepts in Computer Science (WG), volume 5344 of LNCS, pages 300-311, 2008.

[13] J. P. Petersen. Die Theorie der Regulären Graphs. Acta Mathematica, 15:193-220, 1891.

[14] C. Thomassen. Two-coloring the edges of a cubic graph such that each monochromatic component is a path of length at most 5. J. Comb. Theory Ser. B, 75(1):100-109, 1999.

[15] K. Zhu and B. Mukherjee. A review of traffic grooming in WDM optical networks: Architectures and challenges. Optical Networks Magazine, 4(2):55-64, 2003. 


\section{A Strengthening of Proposition 5.4}

In this section, we describe an attempt to prove that the lower bound of $\left\lceil\frac{C+1}{C} \frac{\Delta}{2}\right\rceil$ of Proposition 2.4 is attained in the remaining cases. We attempt to resolve the remaining cases by using induction and Tutte's matching theorem. We may assume that $\Delta$ is odd by Theorem 4.1.

The idea is to use the result from Section 5.2, which solves the case when the graph contains a perfect matching, as a base case for a proof by induction. Then, if the graph does not contain a perfect matching, an easy consequence of Tutte's matching theorem shows that it contains an edge-cut of size at most $\Delta-1$. We would then like to recurse on each side of the cut as we did in the proof of Theorem 3.3 and combine the edge-partitions of each side into a partition of the whole graph. However, as opposed to Theorem 3.3, it is more difficult to deal with the edges across the cut in this case.

We may orient each edge $e=\{u, v\}$ across the cut from $u$ to $v$ and let the side containing $u$ decide which partition will contain $e$. To guarantee that $v$ is not incident to too many subgraphs at the end, we can simply force $v$ to be incident to one fewer subgraph in the edge-partition of the side containing $v$.

We note that, since the cuts have size less than $\Delta$, it is possible to recursively orient the edges of cuts so that no side has more than $\Delta-1$ edges pointing towards it (including edges from previous steps of the recursion).

However, it seems difficult to control the distribution of the edges pointing towards a side. If, for example, a single vertex $v$ had $\Delta-1$ edges pointing towards it, then it is clearly impossible to obtain the desired edge-partition, as $v$ would need to be in a negative number of parts. On the other hand, if it were possible to control the distribution of the edges pointing towards a side, the following strengthening of Proposition 5.4 would be sufficient to prove the base case of the induction.

Definition A.1. $G$ is near- $\Delta$-regular if the vertices of $G$ have degrees between $\frac{\Delta}{2}$ and $\Delta$ and $|E(G)| \geq \frac{\Delta}{2}(|V(G)|-1)-1$ (i.e., the total degree is off by at most $\left.\Delta-1\right)$.

Lemma A.2. Let $L B(C, \Delta)=\left\lceil\frac{C+1}{C} \frac{\Delta}{2}\right\rceil$, the lower bound of Proposition 2.4. Let $C, \Delta$ be positive integers with $\Delta$ odd and $(\Delta-1) / 2$ not a multiple of $C$. Let $G$ be a near- $\Delta$-regular graph with girth at least 5 and a perfect matching. Then $G$ has an edge-partition where each vertex $v$ is incident to at most $L B(C, \Delta)-(\Delta-\operatorname{deg}(v))$ subgraphs of the partition.

We note that it may be possible to first recursively find all the cuts and then orient the edges so that no vertex has more than $(\Delta-1) / 2$ edges pointing towards it. We also note that the above lemma is not in its strongest form (e.g., we could have the total degree differ by more the one stated in the lemma) but we clearly cannot relax the condition that every vertex $v$ has degree greater than $\Delta / 2$ (otherwise, $v$ is contained in too many subgraphs even if we use stars of size $C$ centered at $v$ ). We now prove Lemma A.2.

Proof. Let $M$ be a perfect matching in $G$. Since at most $\Delta$ vertices of $G$ have degree not equal to $\Delta$, at most $\Delta$ edges in $M$ connect an odd degree vertex to an even degree vertex. Let $G^{\prime}$ be the graph obtained from $G$ by removing edges of $M$ matching odd degree vertices of $G$ and adding (at most $\Delta / 2$ ) edges to pair up the remaining odd degree vertices of $G$. Thus, $G^{\prime}$ is an even graph and we may obtain an Eulerian orientation $O^{\prime}$ of $G^{\prime}$.

$O^{\prime}$ induces an orientation of some of the edges of $G$. We orient the remaining edges of $G$ "both ways" and count half towards the in-degree and half towards the out-degree of the vertex. Let $S$ be the set of vertices of $G$ with degree less than $\Delta$. We reverse some of the arcs of $O$ so that all vertices in $S$ have out-degree at least $\Delta / 2$. This can be done greedily since $G$ has no $C_{4}$ and we are only off by $\Delta-1$ from the total degree. We call this new orientation $O$. 
Let $S^{\prime}$ be the set of vertices with out-degree less than $\Delta / 2$ in $O$. Note that the vertices of $S^{\prime}$ have degree $\Delta$ and out-degree at least $(\Delta-3) / 2$. Let $N^{-}(v)$ denote the set of vertices with an arc to $v$ in $O$. Note that for two distinct vertices $u$ and $v$ in $G$, their neighborhood intersects in at most one vertex (since $G$ has girth at least 5). Therefore $N^{-}(u) \cap N^{-}(v)$ also contains at most one vertex.

Therefore, we may find a subgraph $H$ of the graph induced by the edges $N^{-}(v)$ to $v$ for all $v$ in $S^{\prime}$ with the following properties:

- Each vertex in $N^{-}\left(S^{\prime}\right)$ has degree at most 1.

- Each vertex in $s \in S^{\prime}$ has degree at least $\delta^{-}(s)-(\Delta-1) / 2 \geq \delta^{-}(s)-(2 C-1)$, where $\delta^{-}(s)$ is the in-degree of $s$.

We say that a star or double-star is full if it contains exactly $C$ edges.

Now, we can find a set $\mathcal{S}=\mathcal{S}_{1} \cup \mathcal{S}_{2} \cup \mathcal{S}_{3}$ of edge disjoint subgraphs of $G$ such that

- $\mathcal{S}_{1}$ is a set of full double stars centered at the endpoints of unoriented edges in $O$,

- $\mathcal{S}_{2}$ is a set of full stars,

- $\mathcal{S}_{3}=\left\{S_{v}\right\}_{v \in V(G)}$, where $S_{v}$ is a star centered at $v$ of size at most $C-1$, and

- only stars in $\mathcal{S}_{3}$ contain edges of $H$.

These edge disjoint subgraphs can be found greedily by first finding $\mathcal{S}_{1}$ and then partitioning the out-edges of each vertex into sets of size $C$ and a remainder set of edges of size $\leq C$.

Now, for each $s \in S^{\prime}$, remove all but two stars centered at $s$ in $\mathcal{S}_{2}$ and remove one star centered at $s$ in $\mathcal{S}_{3}$. Let $R$ be the set of edges removed in this way. For each edge $e=\{u, v\} \in$ $E(H)$, add an out-edge of $v$ in $R$ if there is any left (and remove this edge from $R$ ) to the star containing $e$. By the properties of $H$, no edges of $R$ are left in the end.

We claim that this new set of subgraphs form a $C$-edge-partition where each vertex $v$ is incident to at most $L B(C, \Delta)-(\Delta-\operatorname{deg}(v))$ partitions.

Indeed, the elements of $\mathcal{S}^{\prime}$ are edge disjoint and have size at most $C$ (since every star in $\mathcal{S}_{3}$ has size at most $C-1$ ). The vertices $v \in V-S^{\prime \prime}$ are incident to

$$
\begin{aligned}
\frac{\Delta+1}{2 C}+\operatorname{deg}(v)-\frac{\Delta+1}{2} & =\frac{\Delta+1}{2 C}+\frac{\Delta-1}{2}-(\Delta-\operatorname{deg}(v)) \\
& =\frac{\Delta+1+C(\Delta-1)}{2 C}-(\Delta-\operatorname{deg}(v)) \\
& =\frac{\Delta(C+1)+1-C}{2 C}-(\Delta-\operatorname{deg}(v)) \\
& \leq L B(C, \Delta)-(\Delta-\operatorname{deg}(v))
\end{aligned}
$$

subgraphs if $v$ is not incident to an unoriented edge, and

$$
\begin{aligned}
1+\left\lceil\frac{\Delta-C}{2 C}\right\rceil+\operatorname{deg}(v)-\frac{\Delta+1}{2} & =1+\left\lceil\frac{\Delta-C}{2 C}\right\rceil+\frac{\Delta-1}{2}-(\Delta-\operatorname{deg}(v)) \\
& =\left\lceil\frac{\Delta+C+C(\Delta-1)}{2 C}\right\rceil-(\Delta-\operatorname{deg}(v)) \\
& =\left\lceil\frac{(C+1) \Delta}{2 C}\right\rceil-(\Delta-\operatorname{deg}(v)) \\
& =L B(C, \Delta)-(\Delta-\operatorname{deg}(v))
\end{aligned}
$$


subgraphs if $v$ is incident to an unoriented edge. This satisfies the conditions in the theorem.

Recall that vertices in $S^{\prime}$ have out-degree at least $(\Delta-3) /(2 C)$. If $v \in S^{\prime}$ and $v$ is incident to an unoriented edge, $v$ appears in

$$
\begin{aligned}
\frac{\Delta-3-(2 C-2)}{2 C}+\operatorname{deg}(v)-\frac{\Delta-3}{2} & =\frac{\Delta-1-2 C}{2 C}+\operatorname{deg}(v)-\frac{\Delta+1}{2}+\frac{4}{2} \\
& =\frac{\Delta-1}{2 C}-2+\operatorname{deg}(v)-\frac{\Delta+1}{2}+2 \\
& \leq \frac{\Delta+1}{2 C}+\operatorname{deg}(v)-\frac{\Delta+1}{2} \\
& \leq L B(C, \Delta)-(\Delta-\operatorname{deg}(v))
\end{aligned}
$$

subgraphs. If $v \in S^{\prime}$ and and $v$ is not incident to an unoriented edge, $v$ appears in

$$
\begin{aligned}
1+\left\lceil\frac{\Delta-3-C-(2 C-2)}{2 C}\right\rceil+\operatorname{deg}(v)-\frac{\Delta-3}{2} & =1+\left\lceil\frac{\Delta-1-C}{2 C}\right\rceil-2+\operatorname{deg}(v)-\frac{\Delta+1}{2}+2 \\
& \leq 1+\left\lceil\frac{\Delta-C}{2 C}\right\rceil+\operatorname{deg}(v)-\frac{\Delta+1}{2} \\
& =L B(C, \Delta)-(\Delta-\operatorname{deg}(v))
\end{aligned}
$$

subgraphs. Again, the conditions in the theorem are satisfied as required. 